Www.jmscr.igmpublication.org

Impact Factor (SJIF): 6.379

Index Copernicus Value: 79.54

ISSN (e)-2347-176x ISSN (p) 2455-0450

crossrefDOI: https://dx.doi.org/10.18535/jmscr/v6i12.176

Journal Of Medical Science And Clinical Research

\title{
Profile of Myocardial Infarction in young patient of age 40 years or below in a tertiary care hospital mainly Tribal based Population
}

\author{
Authors \\ Saikat Sau", Sourav Sau ${ }^{2 *}$, Lina Mukherjee ${ }^{3}$ \\ ${ }^{1}$ Assistant Professor Cardiology, BSMC Bankura, West Bengal \\ ${ }^{2}$ Assistant Professor, Radiotherapy Dept. BMCH Burdwan. West Bengal \\ ${ }^{3}$ Resident at Cardiology, RG KAR MC. Kolkata, West Bengal \\ *Corresponding Author \\ Sourav Sau \\ Assistant Professor, Radiotherapy Dept. BMCH Burdwan, West Bengal, India
}

\begin{abstract}
Objective: To determine the outcome of young AMI patient at index hospitalisation.

Background: Coronary artery disease has a devastating consequence precisely because another wise healthy person in the prime time of life may die or become disabled without prior warning. India now in fourth stage of epidemiological transition where Cardiovascular disease is a leading cause of mortality \& morbidity. Youngs are not immuned. In our study we try to establish the risk factor, presentation, complication in hospital mortality \& outcome of young adults (age <40yrs) in our geographic area after $1^{\text {St }}$ AMI.
\end{abstract}

Method: Total 112 patients admitted in our hospital at B.S.M.C. (Bankura, west bengal) after $1^{\text {st }}$ AMI

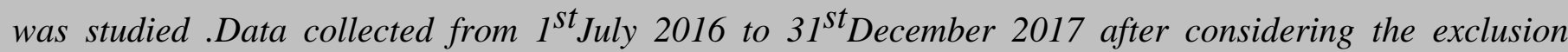
criteria with known ischemic heart disease.

Result: Mean age of presentation was 36.3yrs. Risk factors were smoking, dyslipidaemia, other form of tobacco abuse, and family history of CAD \& Hypertension. Most common presentation was chest pain. Anterior wall was most commonly affected. Young females are comparatively immuned. Presentation was relatively late as it was rural based population \& lack of knowledge. Only twelve patients undergo cardiac catheterisation during index hospitalization.

Conclusions: i) Prognosis is relatively good compare to old age as from other iterature.

ii) Most considerable demonstrated risk factor in young is male sex.

iii) Patient educational status, proper counseling \& availability of resources are important for successful outcome.

\section{Introduction}

There is an increasing trend of coronary artery disease in young patient $<40$ years in Indian population. India is going through an epidemiological tradition where burden of non communicable disease has been rising rapidly. Prevalence of CAD and AMI has progressively been increased in India in last three decades. around $7.1 \%$ in urban and 
$3.7 \%$ in rural population affected with CAD. $\mathrm{CAD}$ in young population deserves special attention as some modifiable risk factors has strong relationship with premature CAD. other than genetic predisposition unhealthy life style such as Cigarette smoking, tobacco use, unbalance dietary pattern, lack of physical activity, effect of unbearable psychological stress added special points with conventional risk factors such as HTN, diabetics and dyslipedemia. Young population also shows a different trend of clinical presentation, risk factor profile and pattern of coronary artery involvement compare to older one. Though the incidence is low but potential for death and long term disability make the entity an important clinical problem.

\section{Material}

Present study was prospective single centre study of 112 patients done at Bankura Sammilani Medical College at Bankura town at Cardiology Department in West Bengal State. The study was conducted on 112 young patients ( $<40$ years) who were admitted with Acute Myocardial infraction over period of 18 months in our department. Patient aged equal or less than 40 years with guideline defined AMI of ACC / AHA were included in the study.

1. AMI was diagnosed according to ACC /AHA guidelines:-

a. New ST elevation at $\mathbf{J}$ point in $>2$ contiguous leads $\geq 2 \mathrm{~mm}$ in men or more than $1.5 \mathrm{~mm}$ in women in lead V2 - V3 and or of $1 \mathrm{~mm}$ in other contiguous chest or limb leads.

b. New or presumably new LBBB was considered as STEMI equivalent. Exclusion criteria was prior $\mathrm{H} / \mathrm{O}$ established $\mathrm{CAD}$, electrolyte abnormality or other non AMI cause of ST elevation. Patient with NSTMI also included in the study.

2. Family history of CAD was defined as any 1 st degree relative younger than 55 years who was affected by ischemic heart disease .
3. Over weight was define by BMI $>25 \mathrm{~kg} / \mathrm{m}^{2}$ and obesity as BMI $>30 \mathrm{~kg} / \mathrm{m}^{2}$.

4. HTN is defined as patient taking antihypertensive drugs or systolic and/or diastolic BP more than $140 / 90$ mmhg.

5.Smoker was define as Patient taking atleast one Bidi or Cigarette per day, Ex-smoker as who quit smoking one month ago. Diabetes was diagnosed by standard guideline of ADA criteria. Hyperlipidemia was define as total plasma cholesterol more than $200 \mathrm{mg} / \mathrm{dl}$ or use of cholesterol lowering drugs. We also considered past history of use of antiischemicdrugs. In female use of oral contraception and male cocaine abuse.

All patients got the standard protocol of treatment as per ACS guideline. Serial ECG done during indoor treatment. Routine blood Bio Chemistry done for all patient. Echocardiography to assess LV function or any other complication Eg. Pericardial effusion, thrombous, Septal rupture. Selective number of patients angiography done as facility is remote from our hospital and transport facility is scares for such critically ill patients.

\section{Result Analysis}

We have studied total 112 patient among them $96 \%$ was male $4 \%$ was female. $40 \%$ of patient population ages $<35$ year and $15 \%$ below ages 30 years. All are physically active. All patients were came from low socioeconomic status (Income $<5000$ Rs per month). They are all manual worker and except female and one male all patient had history of some form of tobacco use/addiction either bidi or khaini. No one had history of any other abuse or cocaine use . No female patient had history of OCP use during their presentation. All female population were married. $8 \%$ of total population shows diabetes either detected during admission or taking medicine for diabetes. Dyslipidemia was found $43 \%$ of population. HTN was found $23 \%$ of population. Among the affected people $17 \%$ shows family H/O premature CAD. Only $4 \%$ of population 
was obese according to standard guideline for detection of obesity

We have studied mainly rural based tribal population. Mean time of presentation was 15.40 hrs from the index event. Chest pain was presentation in $100 \%$ of population, SOB in $6 \%$, Nausea $36 \%$, sweating $87 \%$ palpitation $38 \%$ of population. Thrombolysis done in only $26 \%$ of population. Among them $64 \%$ was Anterior wall, 26\% was inferior wall and rest were NSTMI. In hospital outcome among the patients,68\% developed some form of complication either mechanical or Electrical. Persistent pain more than 48 hrs despite optimal medication found in $2 \%$ of population. LV dysfunction LVEF <45\% (simpson method) developed $54 \%$ of population. Atrial Fibrillation \& Ventriculartachycardia developed 2 percent and 1percent of population during their index hospitalization. Heart Block developed 18 $\%$ of patients of which $12 \%$ required temporary pacing. cardiogenic shock developed $4 \%$ of population. Echocadiographic evidence of significant MR ( $\geq$ grade - II) found in 16 $\%$ of population. Pericardial effusion $34 \%$ of population.

Mortality was $12 \%$ population during index hospitalization. Only twelve patient undergo cardiac catheterisation during index hospital admission period, as cathlab facility is remote from our hospital \& most patient not willing to undergo invasive procedure .

In coronary angiography mostly single vessel coronary artery diseases detected. LAD was the predominent lesion. only six patient undergone PTCA stenting during index hospital admission.

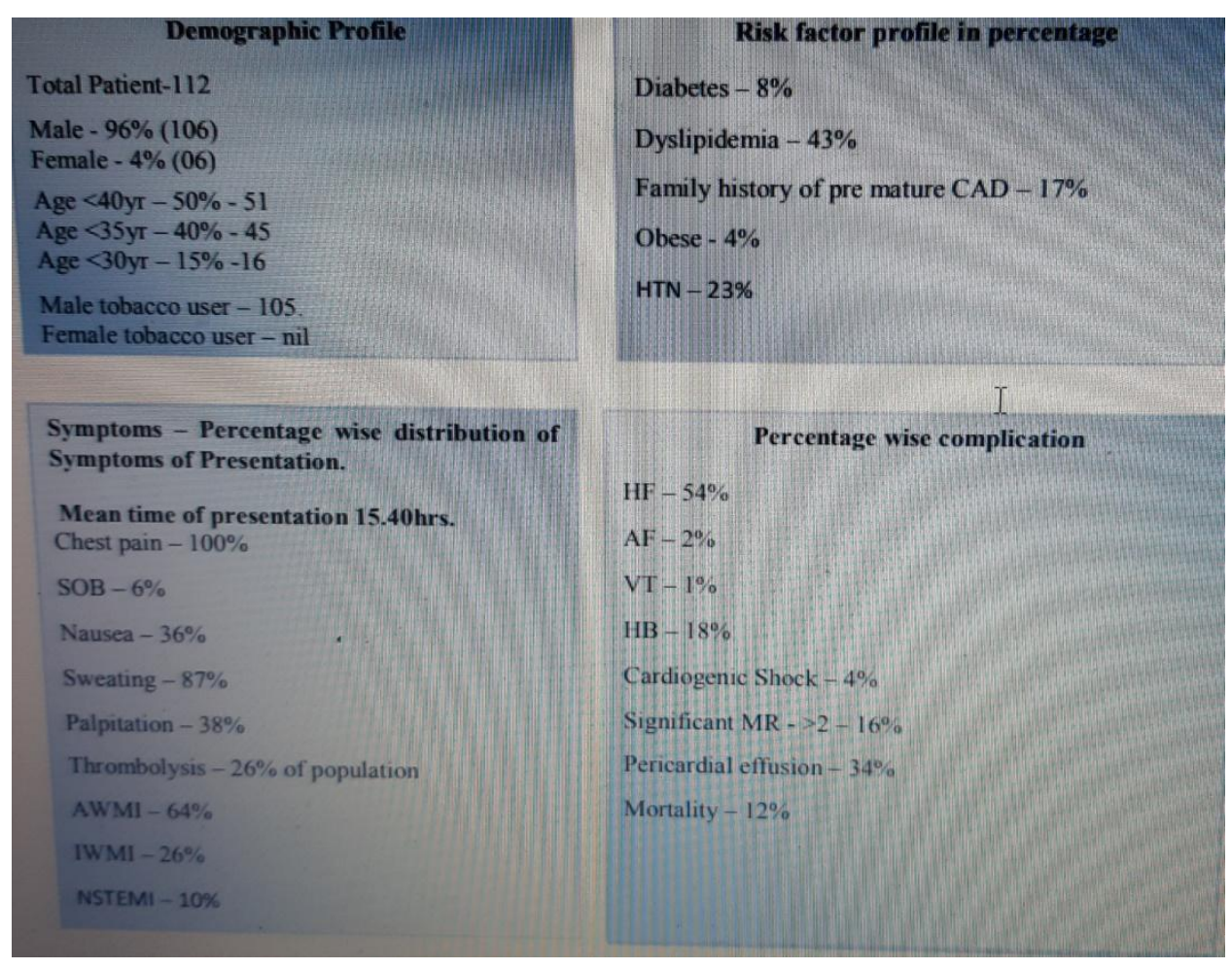

\section{Conclusion}

From the study we came to the following conclusion

1. Young population $<40 \mathrm{yrs}$ are not immune from acute coronary events.

2. Tobacco use and dyslipidemia are important risk factor.
3. Lack of awareness \& facility is a burden to proper treatment protocol.

4. Progonosis is quite good as compared to older age group as stated in other various studies in literature. With rising prevalance of CAD in India world health organisation estimated that by the end of 2020 India will be 
the cardio diabetic capital of the globe. CVD become more prevalent \& aggressive in youngest population as stated in our study. Most considerable demonstrated risk factor in young is male sex . Most common symptoms was chest pain. Female are less preponderance due to probably protected effect of estrogen . Most important modifiable risk factor is smoking \& other form of tobaco use as shown in ourstudy.Dyslipidemia is also a challenging risk factor in our population. Other than Typical angina ,atypical pain , diaphoresis, palpitation, SOB are more common in female .

Prodomal symptoms are also common in younger age group. vulnerable plaque are more common in younger population \& prone to rupture in STEMI patient .CSA are less common in young population. High prevalence of stress are prone to develop rupture. Hypertension are less commom in young population with AMI.

Smoking \& other form of tobacco use are importent risk factor for MI in young population. It adversely affects all phases of atherosclerosis by hastening the thrombotic process, Endothelial dysfunction, coronary vasoconstriction and induces proinflammatory effect.

Smoking cessation should be started as primordial prevention. Obesity is a less important cause of MI in younger population in our study. Central Obesity a component of metabolic syndrome is an important risk factor for ACS in middle age group. Dyslipidemia is also an impartaint risk factor for young MI. low HDLC is maincomponent for development of MI in our study group. As the population reside in remote \& interior places and lack of awareness about the warning symptoms they attend hospital in late.

Mean time of presentation was $15.4 \mathrm{hr}$ after the onset of index event, so window period of thrombolysis could acheived only $26 \%$ of population and no one has contraindication for thrombolysis .Lack of cathlab facility, Financial barrier, health awareness are important Constrain.

\section{References}

1. AMI in very young (aged $\leq 35$ years) Bangladeshi patients:

https://www.sciencedirect.com/science/art icle/pii/S2405587515300238 by M Kalimuddin - 2016

2. Acute myocardial infarction: Clinical features and outcomes in young https://www.ncbi.nlm.nih.gov/pmc/article s/PMC3386311/ by CP Wong - 2012

3. Genetic risk factors in myocardial infarction at young age. - NCBI https://www.ncbi.nlm.nih.gov/pubmed/15 284679 by E Incalcaterra - 2004

4. AMI in young - Indian Perspective Research

Gate https://www.researchgate.net/.../29589371 5_AMI_in_young_-_Indian_Perspective

5. Acute Myocardial Infarction in Women Circulation circ.ahajournals.org/content/circulationah a/133/9/916.full.pdf by LS Mehta - 2016

6. Journal of the American College of Cardiology.Trends in Acute Myocardial Infarction in Young Patients and Differences by Sex and Race, 2001 to 2010.Aakriti Gupta, Yongfei Wang, John A. Spertus, Mary Geda, Nancy Lorenze, Chileshe Nkonde-Price, Gail D'Onofrio, Judith H. Lichtman and Harlan M. Krumholz

7. Sudden Cardiac Arrest in a Young AMI Patient ... - $\quad$ ZOLL LifeVest https://lifevest.zoll.com/sites/default/files/ CSIN_PCICaseStudy_JulyAugust 2012

8. Myocardial infarction in young adults-risk factors and pattern of coronary artery involvement Rajeev Bhardwaj, Arvind Kandoria, Niger Med J. 2014 Jan-Feb; 55(1): 44-47.doi: $\quad 10.4103 / 0300-$ 1652.128161 
9. ARYA Atheroscler. 2017 Mar; 13(2): 7987.Acute myocardial infarction in very young adults: A clinical presentation, risk factors, hospital outcome index, and their angiographic characteristics in North India-AMIYA Study. Santosh Kumar Sinha, 1 Vinay Krishna, 1 Ramesh Thakur, 2 Ashutosh Kumar, 3 Vikas Mishra, 3 Mukesh Jitendra Jha, 3Karandeep Singh, 3 Mohit Sachan, 3 Rupesh Sinha, 3 Mohammad Asif,3 Nasar Afdaali, 3 and Chandra Mohan

10. IOSR Journal of Dental and Medical Sciences (IOSR-JDMS) e-ISSN: 22790853, p-ISSN: 2279- 0861.Volume 15, Issue 1 Ver. VII (Jan. 2016), PP 25-30 www.iosrjournals.org DOI: 10.9790/0853-15172530

www.iosrjournals.org 25 | Page Acute myocardial infarction in young adults of North East India : a clinical and angiographic study Pranab Jyoti Bhattacharyya1 1 (Associate Professor, Department of Cardiology, Gauhati Medical College College/ Srimanta Sankardeva University of Health Sciences, Country India) 